\title{
Microstructural Tissue Changes in Alzheimer Disease Brains: Insights from Magnetization Transfer Imaging
}

\author{
(D). Colonna, (D) M. Koini, (D). Pirpamer, (D)A. Damulina, (D). Hofer, (D). Schwingenschuh, (D). Enzinger, (D) R. Schmidt, and
} (iD) Sopele

\section{ABSTRACT}

BACKGROUND AND PURPOSE: Reductions in magnetization transfer ratio have been associated with brain microstructural damage. We aim to compare magnetization transfer ratio in global and regional GM and WM between individuals with Alzheimer disease and healthy control participants to analyze the relationship between magnetization transfer ratio and cognitive functioning in Alzheimer disease.

MATERIALS AND METHODS: In this prospective study, participants with Alzheimer disease and a group of age-matched healthy control participants underwent clinical examinations and 3T MR imaging. Magnetization transfer ratios were determined in the cortex, AD-signature regions, normal-appearing WM, and WM hyperintensities.

RESULTS: Seventy-seven study participants (mean age \pm SD, $72 \pm 8$ years; 47 female) and 77 age-matched healthy control participants (mean age \pm SD, $72 \pm 8$ years; 44 female) were evaluated. Magnetization transfer ratio values were lower in patients with Alzheimer disease than in healthy control participants in all investigated regions. When adjusting for atrophy and extent of WM hyperintensities, significant differences were seen in the global cortex (OR $=0.47 ; 95 \% \mathrm{Cl}: 0.22,0.97 ; P=.04)$, in Alzheimer disease-signature regions (OR $=0.31 ; 95 \% \mathrm{Cl}: 0.14,0.67 ; P=.003)$, in normal-appearing WM (OR $=0.59 ; 95 \% \mathrm{Cl}: 0.39,0.88 ; P=.01)$, and in WM hyperintensities ( $\mathrm{OR}=0.18 ; 95 \% \mathrm{Cl}: 0.09,0.33 ; P \leq .001$ ). The magnetization transfer ratio in these regions was an independent determinant of $A D$. When correcting for atrophy and WM hyperintensity extent, lower GM magnetization transfer ratios were associated with poorer global cognition, language function, and constructional praxis.

CONCLUSIONS: Alzheimer disease is associated with magnetization transfer ratio reductions in GM and WM regions of the brain. Lower magnetization transfer ratios in the entire cortex and $A D$-signature regions contribute to cognitive impairment independent of brain atrophy and WM damage.

ABBREVIATIONS: $\mathrm{AD}=$ Alzheimer disease; $\mathrm{CERAD}=$ Consortium to Establish a Registry for Alzheimer's Disease; MMSE = Mini-Mental State Examination; MTI = magnetization transfer imaging; MTR = magnetization transfer ratio; NAWM = normal-appearing white matter

A lzheimer disease $(\mathrm{AD})$ represents the most common cause of dementia. Only a few neuroimaging biomarkers have been approved for clinical use, and most are still objects of research. ${ }^{1}$ Although structural MR imaging contributes to the exclusion of other possible causes of a dementia syndrome, brain atrophy measures have only modest sensitivity and specificity for the differential diagnosis of dementia. ${ }^{2}$ The role of

Received July 1, 2020; accepted after revision October 23.

From the Department of Neurology (I.C., M.K., L.P., A.D., P.S., C.E., R.S., S.R.);

Institute for Medical Informatics, Statistics and Documentation (E.H.); and Division of Neuroradiology, Vascular \& Interventional Radiology, Department of Radiology (C.E.), Medical University of Graz, Graz, Austria.

The work originated from the Department of Neurology, Medical University of Graz, Auenbruggerplatz 22, 8036 Graz, Austria.

This study was funded by the Austrian Science Fund (FWF grant No. 12889-B31) and the German Research Foundation (DF DU1626/1-1).

Previously presented at: 6th Congress of the European Academy of Neurology, virtual congress, May 23, 2020.
MR imaging techniques that allow assessment of microstructural brain changes, such as DTI and magnetization transfer imaging (MTI) for detecting AD-related tissue abnormalities, is still widely unknown. Numerous DTI studies have reported loss of WM integrity in $\mathrm{AD}$ and related this to tau accumulation in AD-specific regions. ${ }^{3}$ Only a few studies used MTI to explore microstructural tissue abnormalities in AD.

The magnetization transfer ratio (MTR), which can be derived from MTI, has been shown to be associated with axonal attenuation and myelin content. ${ }^{4-6}$ In patients with $\mathrm{AD}$, MTR reductions were reported in the whole-brain analyses, ${ }^{7-9}$ cortical GM, ${ }^{8,10}$

Please address all correspondence to Reinhold Schmidt, Auenbruggerplatz 22, 8036 Graz, Austria; e-mail: reinhold.schmidt@medunigraz.at

- Indicates open access to non-subscribers at www.ajnr.org

Indicates article with online supplemental data.

http://dx.doi.org/10.3174/ajnr.A6975 
global WM, ${ }^{10}$ hippocampus, ${ }^{7,11,12}$ and temporal lobes. ${ }^{8}$ In a longitudinal study of our own group, patients with $\mathrm{AD}$ had significantly lower global MTR values than control participants. MTR declined significantly over a follow-up period of 12 months and was paralleled by a brain tissue loss of $2.2 \%$ per year. ${ }^{13}$ So far, only a few studies have explored the association between regional MTR changes and cognition in patients with AD. Van der Flier et $\mathrm{al}^{9}$ reported a strong association between whole-brain MTR and global cognitive deterioration in patients with $\mathrm{AD}$, but there was no significant relationship between regional MTR reductions and domain-specific cognitive impairment. In our previous study, we observed direct associations between MTR and Mini-Mental State Examination (MMSE) scores for the hippocampus, putamen, and thalamus. The relationship was stronger in the left than in the right hemisphere. ${ }^{13}$

Here we extend previous work by assessing the role of MTR reductions in the GM and WM in distinguishing patients with mild to moderate $\mathrm{AD}$ from healthy control participants, and we investigate their associations with cognitive decline independent of atrophy and WM damage.

\section{MATERIALS AND METHODS \\ Study Participants}

This prospective study included 77 participants with AD from the longitudinal cohort study Prospective Dementia Registry Austria (PRODEM), ${ }^{14}$ a multicenter study. Current study participants originate exclusively from the Graz center because it was the only center where an MTI sequence had been included in the MR imaging protocol. The study was approved by the ethics committee of the Medical University of Graz, and informed consent was signed by all participants or their caregivers. The data that support the findings of this study are available on request from the corresponding author (R.S.). Dementia was diagnosed according to the Diagnostic and Statistical Manual of Mental Disorders-IV ${ }^{15}$ and National Institute of Neurological and Communicative Disorders and Stroke and the Alzheimer's Disease and Related Disorders Association (NINCDS-ADRDA) criteria. ${ }^{16}$ The severity of dementia was determined according to the MMSE score, as previously described. ${ }^{17}$ A group of 77 agematched healthy control participants was selected from the Austrian Stroke Prevention Study, a large prospective single-center longitudinal study of the healthy older adult population of the city of Graz, Austria, including individuals without neuropsychiatric disease randomly selected from the community register. ${ }^{18}$ All participants underwent a comprehensive clinical evaluation and 3T MR imaging on the same scanner with identical acquisition parameters. The cognitive function of the participants with $\mathrm{AD}$ was assessed with the $\mathrm{MMSE}^{19}$ and the Consortium to Establish a Registry for Alzheimer Disease (CERAD) ${ }^{20}$ test battery.

\section{Image Acquisition}

MR imaging was performed on a 3T whole-body MR system (Tim Trio, Siemens) with a 12-channel head coil. The MR imaging protocol included a T2 FLAIR sequence (TR/TE/ $\mathrm{TI}=10,000 / 69 / 2500 \mathrm{~ms}$, number of slices $=40$, section thickness $=3 \mathrm{~mm}$, in-plane resolution $=0.86 \mathrm{~mm} \times 0.86 \mathrm{~mm})$, a T2-weighted sequence with 2 echoes (TE1/TE2/TR $=10 / 72$ /
$5260 \mathrm{~ms}$, number of slices $=40$, section thickness $=3 \mathrm{~mm}$, inplane resolution $=0.86 \mathrm{~mm} \times 0.86 \mathrm{~mm}$ ), and a 3D T1-weighted magnetization-prepared rapid acquisition of gradient echo sequence with whole-brain coverage (TR/TE/TI $=1900 / 2.19$ / $900 \mathrm{~ms}$, flip angle $=9^{\circ}$, isotropic resolution $=1 \mathrm{~mm}$ ).

Additionally, MTI was performed with a spoiled 3D gradientecho sequence $\left(\mathrm{TR} / \mathrm{TE}=40 / 7.38 \mathrm{~ms}\right.$, flip angle $=15^{\circ}$, number of slices $=40$, section thickness $=3 \mathrm{~mm}$, in-plane resolution $=$ $0.86 \mathrm{~mm} \times 0.86 \mathrm{~mm}$ ), which was performed twice, with and without a Gaussian-shaped radiofrequency saturation prepulse.

\section{Image Processing}

MTR maps were calculated according to the formula MTR = (Mo-Mss)/Mo, in which Mss and Mo are the signal intensities obtained with and without MT saturation, respectively. For regional assessment of MTR values, the cortical structures were segmented fully automated using FreeSurfer (version 5.3; http:// surfer.nmr.mgh.harvard.edu). The technical details of these procedures are described elsewhere. ${ }^{21,22}$ The volumes of all segmented structures were normalized by the estimated total intracranial volume. A visual quality check was done for each case using an in-house developed bash-script based tool, which provides a graphic summary of the segmentations, overlaid on the T1-weighted image. Of all segmentations provided by FreeSurfer, we analyzed 6 ROIs ( $\mathrm{AD}$-signature regions) that were previously identified as sensitive to early effects of $\mathrm{AD} .{ }^{23}$ These regions included the hippocampus, parahippocampal cortex, cuneus, precuneus, entorhinal cortex, and inferior parietal lobule.

After affine registration with the T1-weighted scan using FSL FLIRT (version 6; https://fsl.fmrib.ox.ac.uk/fsl/fslwiki/FLIRT), regional masks were overlaid on the MT-weighted images. To identify and exclude CSF-contaminated voxels in the registered cortical masks, we performed a mask-segmentation optimization using a semiquantitative T2 map, ${ }^{24}$ which was calculated from the mono-exponential decay of both echoes of the T2-weighted sequence and registered to the T1 sequence. After a histogram analysis of the T2 map for each brain-lobe mask, voxel intensities above the full width at half maximum of the histogram peak were defined as CSF-contaminated voxels and were therefore excluded from further analyses. Finally, median MTR values were extracted from the CSF-corrected brain lobes using FSLSTATS (https://fsl. fmrib.ox.ac.uk/fsl/fslwiki/Fslutils). To assess binary masks of WM hyperintensities, we segmented hyperintense T2 lesions on the FLAIR images with an in-house-developed semiautomatic regiongrowing tool. ${ }^{25}$ A normal-appearing white matter (NAWM) mask was calculated by subtracting the manually segmented WM hyperintensity masks from the total WM mask obtained by FreeSurfer. The extent of WM hyperintensity was assessed according to the Fazekas scale. ${ }^{26}$

\section{Statistical Analysis}

For data analysis, we used SPSS (version 23; IBM). Assumptions of normal distribution were tested with the KolmogorovSmirnov test. For bivariate correlations, we performed the Pearson test and a paired $t$ test, and in case of non-normally distributed samples, a Wilcoxon signed-rank test was used to compare the 2 groups. Z-scores were computed for raw scores of 
MTR and normalized volumes, and they were used in the regression analyses. Logistic regression analyses were performed to correlate the diagnosis of $\mathrm{AD}$ with MTR metrics in the global cortex, AD-signature regions, NAWM, and WM hyperintensities. To assess the associations between MTRs and performance on the MMSE and on CERAD, linear regressions were calculated with cognition as the dependent variable and MTR as the predictor variable. All models were adjusted for age, sex, years of education, normalized regional volumes, and Fazekas score. Brain volume and Fazekas score were considered as covariates to determine if MTRs relate to $\mathrm{AD}$ and to cognitive impairment independent of atrophy and extent of WM hyperintensities. For each regression coefficient, the $95 \%$ confidence interval and the $P$ value were determined. A $P$ value $<.05$ was considered statistically significant.

\section{RESULTS}

\section{Participant Characteristics}

A total of 156 individuals with dementia were confirmed eligible and recruited in the longitudinal cohort study PRODEM in Graz.
We excluded 19 participants who were affected by other causes of dementia and 60 individuals who had not undergone a MR imaging examination with sufficient MTI quality (Fig 1). Seventyseven study participants with $\mathrm{AD}$ were included in our study. They were 30 men and 47 women with a mean age of $72 \pm$ 8 years, ranging from 51 to 87 years. Fifty-five patients had mild AD (MMSE score: range 21-28, mean \pm SD: $23.98 \pm 2.13$ ), and 22 patients were diagnosed with moderate to severe AD (MMSE score: range $14-20$, mean \pm SD: $17.14 \pm 1.86)$. Seventy-seven healthy age-matched individuals ( \pm 1 year) served as control participants. Their mean age was also $72 \pm 8$ years, ranging from 51 to 87 years. All control participants had MMSE scores $\geq 24$ with the exception of 3 individuals who had MMSE scores of 23. None of the control participants had symptoms of dementia or mild cognitive impairment. The comparison of demographics, neuropsychological findings, and MR imaging findings between $\mathrm{AD}$ and healthy control participants are displayed in Table 1. Whereas the distribution of sex was similar in both investigational subsets $(P=.62)$, the study participants with $\mathrm{AD}$ performed significantly worse on the MMSE $(P<.001)$, had lower GM $(P<.001)$ and NAWM $(P=.01)$ volumes, and had higher WM hyperintensity volumes $(P=.02)$. A higher grade of chronic small-vessel disease, indicated by Fazekas grade 2 or 3, was present in the $63.7 \%$ of the patients with $\mathrm{AD}$ and in the $40.3 \%$ of the healthy control participants.

137 study participants with diagnosis of probable or possible $A D$

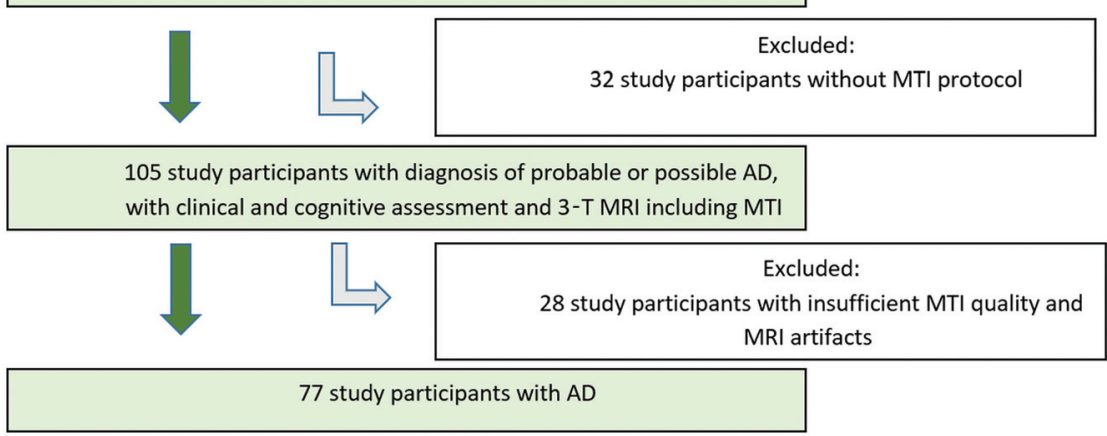

FIG 1. Flowchart shows the recruitment of the study participants with $A D$.

\section{Comparison of MTR Between Patients with AD and Healthy Control Participants}

The study participants with $\mathrm{AD}$ had lower MTR values in the GM $(P<$ $.001)$, NAWM $(P=.003)$, and WM hyperintensities $(P<.001)$ (Table 1$)$. Logistic regression analyses demonstrated that lower MTR values in global cortex $(\mathrm{OR}=0.47 ; 95 \% \mathrm{CI}$ : $0.22,0.97 ; P=.04)$, AD-signature regions $(\mathrm{OR}=0.31 ; 95 \% \mathrm{CI}: 0.14$,

Table 1: Demographic, neuropsychological, and MR imaging findings of study participants

\begin{tabular}{|c|c|c|c|}
\hline Study Participant Characteristic & $\mathrm{AD}(n=77)$ & Healthy Control Participants ( $n=77$ ) & $P$ Value $^{\mathrm{b}}$ \\
\hline No. female (\%) & $47(61)$ & $44(57)$ & .62 \\
\hline Age, years, ${ }^{a}$ & $72(8)$ & $72(8)$ & .98 \\
\hline Age range, years & $51-87$ & $51-87$ & \\
\hline MMSE $^{\mathrm{a}}$ & $22.03(3.72)$ & $27.57(1.75)$ & $<.001$ \\
\hline Global cortex volume, $\mathrm{cm}^{3 \mathrm{a}}$ & $329.63(14.53)$ & $394.04(41.25)$ & $<.001$ \\
\hline AD-signature regions volume, $\mathrm{cm}^{3 \mathrm{a}}$ & $54.81(10.15)$ & $70.28(8.86)$ & $<.001$ \\
\hline Global NAWM volume, $\mathrm{cm}^{3 \mathrm{a}}$ & 305.77 (63.71) & $334.70(52.52)$ & .01 \\
\hline WM hyperintensities volume, $\mathrm{cm}^{3 \mathrm{a}}$ & 16.19 (17.69) & 11.64 (18.01) & .02 \\
\hline Fazekas grade 2 or 3 , no. (\%) & 49 (63.7) & $31(40.3)$ & \\
\hline Global cortex MTRs ${ }^{a}$ & $0.295(0.016)$ & $0.309(0.008)$ & $<.001$ \\
\hline AD-signature regions $M_{T R s^{a}}$ & $0.297(0.018)$ & $0.309(0.009)$ & $<.001$ \\
\hline Global NAWM MTRs ${ }^{a}$ & $0.384(0.009)$ & $0.388(0.009)$ & .003 \\
\hline WM hyperintensities MTRs ${ }^{\mathrm{a}}$ & $0.322(0.028)$ & $0.350(0.016)$ & $<.001$ \\
\hline
\end{tabular}

\footnotetext{
${ }^{a}$ Data are mean \pm SDs.
}

${ }^{b}$ Pearson $\chi 2$ for nominal, Wilcoxon, and paired $t$ test for continuous variables were applied. 
$0.67 ; P=.003)$, NAWM $(\mathrm{OR}=0.59 ; 95 \% \mathrm{CI}: 0.39,0.88 ; P=.01)$, and WM hyperintensities ( $\mathrm{OR}=0.18 ; 95 \% \mathrm{CI}: 0.09,0.33$; $P \leq .001)$ remained significantly related to $\mathrm{AD}$ even after correction for age, sex, and years of education as well as brain atrophy and extent of WM hyperintensities (Table 2). When considering the $\mathrm{AD}$-signature regions individually, we found that decreased MTR values in the parahippocampal cortex $(\mathrm{OR}=0.61 ; 95 \% \mathrm{CI}$ : $0.37,0.99 ; P=.04)$, cuneus (OR $=0.26$; $95 \% \mathrm{CI}: 0.09,0.73 ; P=$ $.01)$, precuneus (OR $=0.24 ; 95 \% \mathrm{CI}: 0.12,0.45 ; P \leq .001)$, and entorhinal cortex $(\mathrm{OR}=0.57$; 95\% CI: 0.35, 0.95; $P=.03$ ) were associated with $\mathrm{AD}$ diagnosis (Online Supplemental Data). Furthermore, we found that reduced MTRs in NAWM and WM hyperintensities were related to $\mathrm{AD}$ independent of dementia severity; by contrast, in only the patients with mild $\mathrm{AD}$, lower MTRs in the $\mathrm{AD}$-signature regions $(\mathrm{OR}=0.38,95 \% \mathrm{CI}$ : 0.17 , $0.82 ; P=.02)$ were associated with AD.

\section{MTR and Cognition in AD}

Table 3 displays the associations between MTRs and cognitive functioning in the AD cohort. Only GM MTRs were significantly related to cognitive measures; no such relationship existed for WM MTRs. When adjusting for age, sex, education, normalized regional volume, and Fazekas score, lower MTRs in the global cortex were related to lower scores on the language subtest of the CERAD test battery ( $\beta=0.31, P=.02$ ). Only a nonsignificant trend was seen for the association between cortical MTR and MMSE and between global MTRs in the AD-signature regions and poorer performance on CERAD subtests for language function and constructional praxis. However, when considering the AD-signature regions individually, lower MTRs in the cuneus ( $\beta=0.30$; 95\% CI: $0.26,1.95 ; P=.01)$ and hippocampus $(\beta=$ 0.29 ; $95 \%$ CI: $0.03,2.22 ; P=.04$ ) were significantly related to a poorer performance on the MMSE. With regard to the CERAD test, reduced MTRs in the parahippocampal cortex $(\beta=0.38$; 95\% CI: $0.28,1.41 ; P=.006$ ), cuneus ( $\beta=0.45$; 95\% CI: 0.51 , 1.56; $P \leq .001)$, and entorhinal cortex ( $\beta=0.35$; 95\% CI: 0.25 ,

Table 2: Logistic regression analysis-lower MTRs relate to AD independent of normalized regional brain volume and white matter damage

\begin{tabular}{lccc}
\hline \multicolumn{1}{c}{ MTR Region } & OR & 95\% Cl & $\boldsymbol{P ~ V a l u e ~}^{\mathrm{a}}$ \\
\hline Global cortex & 0.47 & $0.22-0.97$ & .04 \\
AD-signature regions & 0.31 & $0.14-0.67$ & .003 \\
Global NAWM & 0.59 & $0.39-0.88$ & .01 \\
WM hyperintensities & 0.18 & $0.09-0.33$ & $<.001$ \\
\hline
\end{tabular}

${ }^{a}$ Corrected for age, sex, years of education, normalized regional volume, and Fazekas score.
1.28; $P=.004)$ were associated with worse constructional praxis (Online Supplemental Data). There existed no significant associations with other CERAD subtests. No effect of laterality on the MTR correlations with poor language function was found (data not shown).

\section{DISCUSSION}

Our study shows lower MTRs in cortical and subcortical structures, including $\mathrm{AD}$-signature regions, in NAWM, and WM hyperintensities in patients with AD. Lower WM MTRs were related to $\mathrm{AD}$ diagnosis in patients with both mild and moderately severe $\mathrm{AD}$. However, MTRs in the $\mathrm{AD}$-signature regions were related to $\mathrm{AD}$ diagnosis only in study participants with mild disease. One explanation for these discrepant associations between GM MTRs in mild versus moderately severe AD might be that GM microstructural changes are indeed an early disease phenomenon. Yet we cannot exclude that the small sample size of patients with severe disease and resulting low statistical power are responsible for the lack of a significant association between MTR in GM regions and AD diagnosis. With regard to cognition, only GM but none of the WM MTR measures were related to cognitive functioning in patients with $\mathrm{AD}$. The effects of MTR reductions in the cortex and in the $\mathrm{AD}$-signature regions on global cognition, language function, and constructional praxis were modest, but they were independent of brain atrophy and WM damage. Previous work also reported reduced MTRs in the cortex, ${ }^{8,10}$ hippocampus, ${ }^{7,27}$ and $\mathrm{WM}^{10,28}$ in $\mathrm{AD}$. So far, only a limited number of studies have examined the relationship between MTR values and cognition in individuals with AD. ${ }^{7,9,10,29}$ In the current work, reduced MTRs in the GM were associated with worse global cognition, language function, and constructional praxis. Van der Flier et $\mathrm{al}^{9}$ reported an association between poorer cognitive performance and lower peak heights of the MTR histograms in the whole brain and frontal and temporal lobes. Here we extend this previous study by demonstrating that the association between MTR and cognition in AD exists particularly with cortical MTR and that it is independent of brain atrophy. Like others, ${ }^{7,10,29}$ we failed to observe a relationship between WM MTR and cognition in AD. We realize that we cannot rule out with certainty that reductions in MTR might have been caused by partial volume effects from the CSF as a consequence of atrophy in patients with dementia. CSF has very low MTR values, and sulcal enlargement could cause a reduction of MTR in voxels because of the combination of cortex and CSF. ${ }^{30}$ However, we do not believe that partial volume effects had a considerable effect in our study because cortical volume was considered as a

Table 3: Linear regression analysis in AD-cortical MTRs relate to poorer language function in patients with AD

\begin{tabular}{|c|c|c|c|c|c|c|c|c|c|}
\hline \multirow[b]{2}{*}{ MTR Region } & \multicolumn{3}{|c|}{ MMSE, $n=77$} & \multicolumn{3}{|c|}{$\begin{array}{l}\text { Language Function (CERAD Test: } \\
\text { Boston Naming Test), } n=77\end{array}$} & \multicolumn{3}{|c|}{$\begin{array}{l}\text { Constructional Praxis (CERAD } \\
\text { Test: Figure Copying), } n=73\end{array}$} \\
\hline & $\boldsymbol{\beta}$ & $95 \% \mathrm{Cl}$ & $P$ Value $^{\mathrm{a}}$ & $\beta$ & $95 \% \mathrm{Cl}$ & $P$ Value $^{\mathrm{a}}$ & $\boldsymbol{\beta}$ & $95 \% \mathrm{Cl}$ & $P$ Value $^{\mathrm{a}}$ \\
\hline Global cortex & 0.23 & $-0.07-1.96$ & .06 & 0.31 & $0.19-1.95$ & .02 & 0.22 & $-0.09-1.24$ & .09 \\
\hline AD-signature regions & 0.14 & $-0.50-1.52$ & .32 & 0.28 & $-0.02-1.74$ & .05 & 0.29 & $-0.01-1.32$ & .05 \\
\hline Global NAWM & 0.17 & $-0.24-1.67$ & .14 & 0.11 & $-0.05-1.23$ & .40 & 0.23 & $-0.05-1.23$ & .07 \\
\hline WM hyperintensities & 0.01 & $-0.78-0.88$ & .91 & -0.01 & $-0.81-0.67$ & .89 & 0.01 & $-0.53-0.59$ & .92 \\
\hline
\end{tabular}

Note: $-\beta$ indicates standardized regression coefficient

${ }^{a}$ Corrected for age, sex, years of education, normalized regional volume, and Fazekas score. 
confounder, and all MTR masks have been corrected by deleting voxels with high intensities in the co-registered pseudo-T2 map to prevent CSF contamination of cortical MTR. However, the histopathologic correlates of MTR reductions in the cortex in AD are widely unknown. Decreased MTRs caused by focal demyelination in the cortex were found in patients with multiple sclerosis, ${ }^{31}$ but it is unlikely that loss of cortical myelin is responsible for lowering of cortical MTRs in those with AD. A more likely explanation comes from a study of Patel and coworkers, ${ }^{32}$ who found that dendritic branching is likely to be the main driver of MTR values in the cortex because higher surface area of cellular membranes relates to greater magnetization transfer between the free water and bound water pool. The authors indicated that dendrites and their spines contribute 34 -fold more exposure to the extracellular water than myelin and concluded that MTRs in the cortex are more sensitive to cellular membranes associated with dendrites than myelin. ${ }^{32}$ The loss of dendritic spines directly correlates with the loss of synaptic function. Patients with $\mathrm{AD}$ show a significant loss of synapses and their cognitive capabilities correlate strongly with synapse attenuation. ${ }^{33}$

Another possible explanation for MTR lowering in AD brains is posttranslational modification of axonal proteins. This is suggested by an MTI study on unfixed postmortem brain slices, which assessed blocks containing microscopically nonlesional brain tissue and microdissected adjacent tissue to quantify specific protein levels. ${ }^{34}$ The authors reported that lowering of MTR was caused by a hyperphosphorylation-related change in proton mobility. In line with this finding, altered cortical MTRs may thus reflect hyperphosphorylation of proteins such as tau and pathologic protein accumulation, a process that is known to precede cell death in $\mathrm{AD}$ and other primary neurodegenerative diseases. ${ }^{35}$ The mentioned mechanisms are in keeping with the main finding of our study that MTR changes in the cortex in patients with $\mathrm{AD}$ are at least partly independent of cortical volume and that cortical MTR reductions explain cognitive impairment beyond regional cortical atrophy.

Our study has several strengths. We investigated a considerably large sample of individuals with $\mathrm{AD}$ and of healthy community-dwelling older adults. The availability of a structural scan with high resolution allowed segmentation of cortical and WM compartments. One limitation of our study is that we used the 1984 NINCDS-ADRDA criteria for the diagnosis of AD. ${ }^{16}$ These criteria were standard at the time of patient recruitment into the study. Recently, the criteria have been revised, yet it was shown that the 1984 criteria provide a diagnostic sensitivity and specificity of $81 \%$ and $70 \%$, respectively, in clinicopathologic studies. ${ }^{36}$ Another limitation was that the spatial resolution of the MT sequence was limited, and therefore partial volume effects caused by CSF in cortical regions cannot be ruled out with certainty. However, these effects should be small, and they have been considered in image postprocessing and in the statistical analyses by correcting for atrophy.

\section{CONCLUSIONS}

MTR values were reduced not only in the GM but also in the WM of patients with AD. Only the MTR values in the GM were related to cognitive impairment. At this point, the diagnostic contribution of MTR in patients with AD is still unknown, but our data indicate that it provides additional information beyond pure assessment of brain atrophy and WM damage. How ADrelated pathophysiology can affect the MT mechanism in the GM is not fully determined. Moreover, longitudinal studies in the prodromal stages of $\mathrm{AD}$ are needed to evaluate if MTR is able to add to the prediction of conversion to $\mathrm{AD}$.

Disclosures: Reinhold Schmidt-UNRELATED: Consultancy: AXON Neuroscience; Grants/Grants Pending: Austrian Science Fund.* *Money paid to institution.

\section{REFERENCES}

1. Bayram E, Caldwell JZK, Banks SJ. Current understanding of magnetic resonance imaging biomarkers and memory in Alzheimer's disease. Alzheimers Dement (N Y) 2018;4:395-413 CrossRef Medline

2. Mahalingam S, Chen MK. Neuroimaging in dementias. Semin Neurol 2019;39:188-99 CrossRef Medline

3. Strain JF, Smith RX, Beaumont H, et al. Loss of white matter integrity reflects tau accumulation in Alzheimer disease defined regions. Neurology 2018;91:E313-18 CrossRef Medline

4. Schmierer K, Scaravilli F, Altmann DR, et al. Magnetization transfer ratio and myelin in postmortem multiple sclerosis brain. Ann Neurol 2004;56:407-15 CrossRef Medline

5. Mottershead JP, Schmierer K, Clemence M, et al. High field MRI correlates of myelin content and axonal density in multiple sclerosis: a post-mortem study of the spinal cord. J Neurol 2003;250: 1293-1301 CrossRef Medline

6. Schmierer K, Tozer DJ, Scaravilli F, et al. Quantitative magnetization transfer imaging in postmortem multiple sclerosis brain. $J$ Magn Reson Imaging 2007;26:41-51 CrossRef Medline

7. Ridha BH, Symms MR, Tozer DJ, et al. Magnetization transfer ratio in Alzheimer disease: comparison with volumetric measurements. AJNR Am J Neuroradiol 2007;28:965-70 Medline

8. Bozzali M, Franceschi M, Falini A, et al. Quantification of tissue damage in AD using diffusion tensor and magnetization transfer MRI. Neurology 2001;57:1135-37 CrossRef Medline

9. van der Flier WM, van den Heuvel DMJ, Weverling-Rijnsburger AWE, et al. Cognitive decline in AD and mild cognitive impairment is associated with global brain damage. Neurology 2002;59: 874-79 CrossRef Medline

10. Kabani NJ, Sled JG, Chertkow H. Magnetization transfer ratio in mild cognitive impairment and dementia of Alzheimer's type. NeuroImage 2002;15:604-10 CrossRef Medline

11. Hanyu H, Asano T, Iwamoto T, et al. Magnetization transfer measurements of the hippocampus in patients with Alzheimer's disease, vascular dementia, and other types of dementia. AJNR Am J Neuroradiol 2000;21:1235-42 Medline

12. Hanyu H, Asano T, Sakurai $H$, et al. Magnetisation transfer measurements of the subcortical grey and white matter in Parkinson's disease with and without dementia and in progressive supranuclear palsy. Neuroradiology 2001;43:542-46 CrossRef Medline

13. Ropele S, Schmidt R, Enzinger C, et al. Longitudinal magnetization transfer imaging in mild to severe Alzheimer disease. AJNR Am J Neuroradiol 2012;33:570-75 CrossRef Medline

14. Seiler S, Schmidt H, Lechner A, et al. Driving cessation and dementia: results of the Prospective Registry on Dementia in Austria (PRODEM). PLoS One 2012;7:e52710 CrossRef Medline

15. American Psychiatric Association. Section II: Diagnostic Criteria and Codes. Diagnostic and Statistical Manual of Mental Disorders. 5th edition. American Psychiatric Association 2013

16. McKhann G, McKhann G, Drachman D, et al. Clinical diagnosis of Alzheimer's disease: report of the NINCDS-ADRDA Work Group under the auspices of Department of Health and Human Services Task Force on Alzheimer's Disease. Neurology 1984;34:939-44 CrossRef Medline 
17. National Institute for Health and Clinical Excellence. Donepezil, Galantamine, Rivastigmine, and Memantine for the Treatment of Alzheimer's Disease. National Institute for Health and Clinical Excellence 2011. https://www.nice.org.uk/guidance/ta217/resources/donepezilgalantamine-rivastigmine-and-memantine-for-the-treatment-ofalzheimers-disease-pdf-82600254699973. Accessed September 14, 2020

18. Schmidt R, Lechner H, Fazekas F, et al. Assessment of cerebrovascular risk profiles in healthy persons: definition of research goals and the Austrian Stroke Prevention Study (ASPS). Neuroepidemiology 1994;13:308-13 CrossRef Medline

19. Folstein MF, Folstein SE, McHugh PR. "Mini-mental state.” A practical method for grading the cognitive state of patients for the clinician. J Psychiatr Res 1975;12:189-98 CrossRef Medline

20. Morris JC, Heyman A, Mohs RC, et al. The consortium to establish a registry for Alzheimer's disease (CERAD). Part I. Clinical and neuropsychological assessment of Alzheimer's disease. Neurology 1989;39:1159-65 CrossRef Medline

21. Fischl B, Sereno MI, Dale AM. Cortical surface-based analysis. I. Segmentation and surface reconstruction. NeuroImage 1999;9:195207 CrossRef Medline

22. Reuter M, Rosas HD, Fischl B. Highly accurate inverse consistent registration: a robust approach. NeuroImage 2010;53:1181-96 CrossRef Medline

23. Wu A, Sharrett AR, Gottesman RF, et al. Association of brain magnetic resonance imaging signs with cognitive outcomes in persons with nonimpaired cognition and mild cognitive impairment. JAMA Netw Open 2019;2:e193359 CrossRef Medline

24. Petrovic A, Scheurer E, Stollberger R. Closed-form solution for T2 mapping with nonideal refocusing of slice selective CPMG sequences. Magn Reson Med 2015;73:818-27 CrossRef Medline

25. Plummer DL. DispImage: a display and analysis tool for medical images. Rivista di Neuroradiologia 1992;5:489-95 CrossRef

26. Fazekas F, Chawluk JB, Alavi A. MR signal abnormalities at $1.5 \mathrm{~T}$ in Alzheimer's dementia and normal aging. AJNR Am J Neuroradiol 1987;8:421-26 CrossRef Medline
27. Hanyu H, Asano T, Sakurai H, et al. Magnetization transfer measurements of the hippocampus in the early diagnosis of Alzheimer's disease. J Neurol Sci 2001;188:79-84 CrossRef Medline

28. van Es ACGM, van der Flier WM, Admiraal-Behloul F, et al. Lobar distribution of changes in gray matter and white matter in memory clinic patients: detected using magnetization transfer imaging. AJNR Am J Neuroradiol 2007;28:1938-42 CrossRef Medline

29. van Es ACGM, van der Flier WM, Admiraal-Behloul F, et al. Magnetization transfer imaging of gray and white matter in mild cognitive impairment and Alzheimer's disease. Neurobiol Aging 2006;27:1757-62 CrossRef

30. Seiler S, Pirpamer L, Hofer E, et al. Magnetization transfer ratio relates to cognitive impairment in normal elderly. Front Aging Neurosci 2014;6:263 CrossRef Medline

31. Chen JTH, Easley K, Schneider C, et al. Clinically feasible MTR is sensitive to cortical demyelination in MS. Neurology 2013;80:24652 CrossRef Medline

32. Patel Y, Shin J, Gowland PA, et al. Maturation of the human cerebral cortex during adolescence: myelin or dendritic arbor? Cereb Cortex 2019;29:3351-62 CrossRef Medline

33. Dorostkar MM, Zou C, Blazquez-Llorca L, et al. Analyzing dendritic spine pathology in Alzheimer's disease: problems and opportunities. Acta Neuropathol 2015;130:1-19 CrossRef Medline

34. Petzold A, Tozer DJ, Schmierer K. Axonal damage in the making: neurofilament phosphorylation, proton mobility and magnetisation transfer in multiple sclerosis normal appearing white matter. Exp Neurol 2011;232:234-39 CrossRef Medline

35. Nakamura T, Lipton SA. Cell death: protein misfolding and neurodegenerative diseases. Apoptosis 2009;14:455-68 CrossRef Medline

36. Knopman DS, DeKosky ST, Cummings JL, et al. Practice parameter: diagnosis of dementia (an evidence-based review): Report of the quality standards subcommittee of the American Academy of Neurology. Neurology 2001;56:1143-53 CrossRef Medline 